\title{
Going Beyond the Big Three of Calcutta Physicists: B B Ray, D M Bose and S K Mitra
}

\author{
Rajinder Singh*
}

(Received 31 March 2018; revised 23 April 2018)

\begin{abstract}
C V Raman, S N Bose and M N Saha are the three big physicists of Calcutta who are wellknown. Scientific achievements of other three scientists of Calcutta, namely B B Ray (1894-1944), D M Bose (1885-1975) and S K Mitra (1890-1963), were of international level. For their scientific work, all the three were elected as Fellows of the Indian National Science Academy. The attainments of the three are less known to the physics community. This communication presents a brief review about their scientific achievements.
\end{abstract}

Key words: B B Ray, C V Raman, D M Bose, Indian National Science Academy, Nobel Prize, Royal Society London, S K Mitra.

\section{INTRODUCTION}

B B Ray, S K Mitra and D M Bose (Fig. 1) belonging to 'Calcutta School of Physics' did some pioneering and excellent contributions. All the three were elected Fellow of the India National Science Academy. A short review of their lives and scientific works will give an idea how they have influenced different fields of researches. ${ }^{1}$

\section{Bidhu Bhushan Ray - A Pioneer of X-ray Absorption Spectroscopy}

B B Ray ${ }^{2}$ (BBR) started his research career under C V Raman, who was Palit Professor of Physics at the University of Calcutta. BBR's D.Sc. thesis was evaluated by the British scientists, G C Simpson, J W Nicholson and E H Barton. On $1^{\text {st }}$ November, 1922, they submitted the following report:

We have examined the thesis submitted by $\mathrm{Mr}$. Bidhubushan Ray, M.Sc., and consider that the work described in these is of sufficient merit to
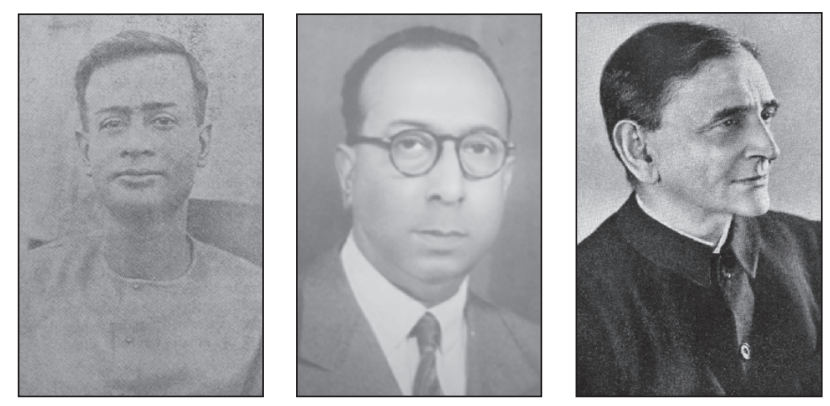

Fig. 1. (Left to Right) B B Ray (Credit: Science and Culture). S K Mitra and D M Bose (Courtesy: University of Calcutta).

warrant the conferment upon Mr. Bidhubushan Ray of the Doctorate. The main thesis entitled 'The scattering of light by liquid droplets and the theory of coronas, glories and iridescent clouds' deals with an interesting branch of meteorological optics and the results obtained by Mr. Bidhubushan Ray are new and valuable (Minutes of the Syndicate, 1923, p. 320).

In the beginning of the 1920s, Ray went to Europe for higher studies and worked in the laboratories of Manne Siegbahn at Stockholm; and

\footnotetext{
* Research Group: Physics Education and History of Science, Department of Physics, Physics Institute, University of Oldenburg, 26111 Oldenburg, Germany. E-Mail: rajinder.singh@uni-oldenburg.de

1 Singh, 2014; Singh, 2016b; Roy \& Singh, 2017; Singh, 2017a.

2 A few short articles dealing with B B Ray's life are Sen, 1944; Anonymous, 1944-1945; 1944; http://insaindia.res.in/ deceaseddetail.php?id=N350654, Oct. 31, 2016; Singh, 2017d; 2017 e.
} 
Niels Bohr at Copenhagen. In the middle of 1926 he returned back to India. It was only with the support of Bohr and Raman that he received proper research facilities at the University of Calcutta (Singh \& Riess, 2001; Singh, 2017a, pp. 73-84). He continued work on X-ray spectroscopy under difficult conditions. In 1930, he found an interesting effect while working on scattering of $\mathrm{X}$-rays. Scientists in the USA and Germany were unable to reproduce his results which led to a controversy. Unsuccessfully, Ray sought support from N Bohr. In 1936, the controversy was resolved by the German physicist, Arnold Sommerfeld, who proved theoretically the correctness of Ray's results (Singh, 2017b).

Almost at the end of his life Ray was able to establish a laboratory at the University of Calcutta. He and his students published a number of articles on X-ray absorption spectroscopy. However, Ray's scientific work did not get due recognition due to lack of application of X-ray absorption spectroscopy, and Ray's premature death in 1944 at the age of 50. Due to computers and the application of Fourier analysis, impetus to the field came in the 1970s (van Bokhoven \& Lamberti, 2016, pp. 3-22).

One of the interesting aspects of Ray's life was his contact with the Indian politician and freedom fighter Netaji Subhash Chandra Bose. They were classmate. Ray is supposed to be the man, who established Bose's contact with the Germany dictator Adolf Hitler, through Werner Heisenberg, one of the founders of the quantum mechanics (Singh, forthcoming).

In the well-known Saha-Raman controversy regarding the leadership of the IACS, B B Ray supported M N Saha in spite of the fact that at different occasions, Raman supported his student Ray (Singh, 2017a).
B B Ray married Asha Sen, whose father Nishi Kanto Sen was Registrar at the University of Delhi. Their daughter Uma Sen was brought up by her mother. She (Uma Sen) lives at the Viswabharati University in Santiniketan.

\section{Sisir Kumar Mitra - A Pioneer of IONOSPheric- AND RAdio Physics}

A few short articles deal with S K Mitra's life and work. ${ }^{3}$ Like B B Ray, S K Mitra also started his research career under C V Raman. Lesser known fact is that Mitra gave a new geometrical theory of the diffraction-figures observed in the heliometer (Mitra, 1920). In 1920, he went to France for further studies; and worked in the laboratories of Charles Fabry and Marie Sklodowska-Curie. There he received his second D.Sc. degree, on the basis of the thesis 'Determination of spectroscopic standard wave-lengths in the short wave-length region' (Mitra, 1923), as was reported in the Journal of Chemical Society (Anonymous, 1923). In France, Mitra changed field of research. He worked with the French radio communication scientist Camille Gutton. Before leaving for India, he (Mitra) wrote a letter to Sir Asutosh Mukherjee, and revealed his plan to initiate wireless technology at the University of Calcutta (Ghosh \& Ghosh, 1995).

Mitra is known for: a) establishing the Radio Physics and the Radio Research Board; b) starting ionospheric science, in particular the discovery of the D-layer and explanation of the E-layer; c) giving theory of active nitrogen in the atmosphere and d) writing a landmark book The upper atmosphere.

For the above achievements, in 1958, he was elected as a Fellow of the Royal Society (FRS), London (Singh, 2017c). S K Mitra was associated with the Indian Science Congress,

\footnotetext{
${ }^{3}$ For various aspects of S K Mitra's life and work, see, Ratcliffe, 1964; INSA, 1994, pp. 501-502; Mahanti, 2003; Das Gupta, 2000; Ramanathan, 1967; Bose, 2010.
} 


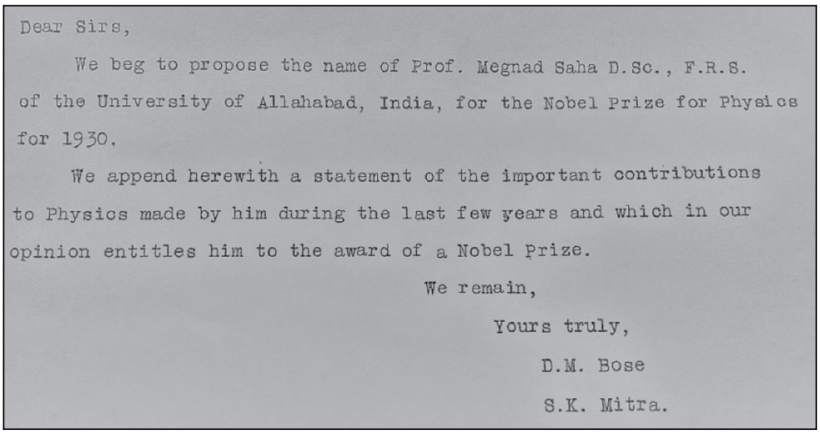

Fig. 2. A part of nomination letter by D M Bose and S K Mitra to the Nobel Committee. (Credit: Swedish Royal Society, Stockholm).

Indian National Science Academy, Indian Association for the Cultivation of Science, University of Calcutta, Asiatic Society of Bengal, and many more institutions. Along with D M Bose, Mitra was the first Indian physicist to be asked by the Nobel Committee to nominate candidates. Both of them, in a joint letter, nominated M N Saha for the Physics Nobel Prize for the year 1930 (Fig. 2).

C V Raman being Nobel Laureate had permanent right to propose candidates. After him, Mitra was the second Indian physicist who got more than three invitations from the Nobel Committee. Again and again, he nominated M N Saha; and even sought support from British and American physicists. However, he was not successful in his endeavour. Except in 1930, the Nobel Committee never asked its expert to write report on Saha's achievements (Singh, 2016a, pp. $38-61)$.

\section{D M Bose - A Multi-talented SCIENTIST}

D M Bose (DMB) belongs to the elite scientists of India. ${ }^{4}$ He was nephew of the legendry physicist J C Bose. The family had close contact with the Nobel Laureate Rabindranath Tagore.
DMB communicated with renowned physicists like N Bohr, M Planck, R A Millikan, J J Thomson and A Einstein. Lesser known fact is that DMB was instrumental in motivating $M$ N Saha and $S$ $\mathrm{N}$ Bose in translating the works of A Einstein and $\mathrm{H}$ Minkowski from German in English, which was published by the University of Calcutta under the title 'The Principle of Relativity'. Einstein's letter of Jan. 26, 1920, written to D M Bose shows that permission was granted to publish the book by Einstein as he did not see any reason that his European publisher will oppose the Indian publication. As it turned out later, Einstein was mistaken. Due to objection by the European publisher to sell this book outside India, Einstein wrote a letter to DMB in July 1922, which he never received. In the letter of March 7, 1923, Bose thanked Einstein for the letter of January 28, 1923. He told Einstein that the University of Calcutta informed its distributor to send back the copies from U.K.

DMB studied abroad under the renowned British physicist $\mathrm{J} \mathrm{J}$ Thompson, the discoverer of electron. In 1914, at the newly found College of Science and Technology, University of Calcutta, he was appointed as Rashbehary Ghosh Professor of Physics. Shortly after that he went to Berlin for higher studies. There he improved Wilson Cloud Chamber and made observation of radioactive radiation. He did $\mathrm{PhD}$ under the guidance of the renowned physicists $\mathrm{H}$ Rubens and M Planck (Fig. 3). After his return from Berlin, in Calcutta he established a research group to study radioactivity with cloud chamber, which was indigenously built by him.

D M Bose was known for: a) establishing Cloud Chamber Research in Calcutta; b) 'Bose theory', 'Bose-Stoner theory' or 'Welo-Bose rules' for paramagnetic substances. With his associates he discovered a new photo-magnetic effect, which

\footnotetext{
A few short articles dealing with D M Bose's life are Roy, 2010; Burma, 1981; B.I. 1955; INSA, 1994; Mukherji, 2015; Chatterjee, 1975.

5 For more detail, see, Singh, 201b6, pp. 35-74.
} 


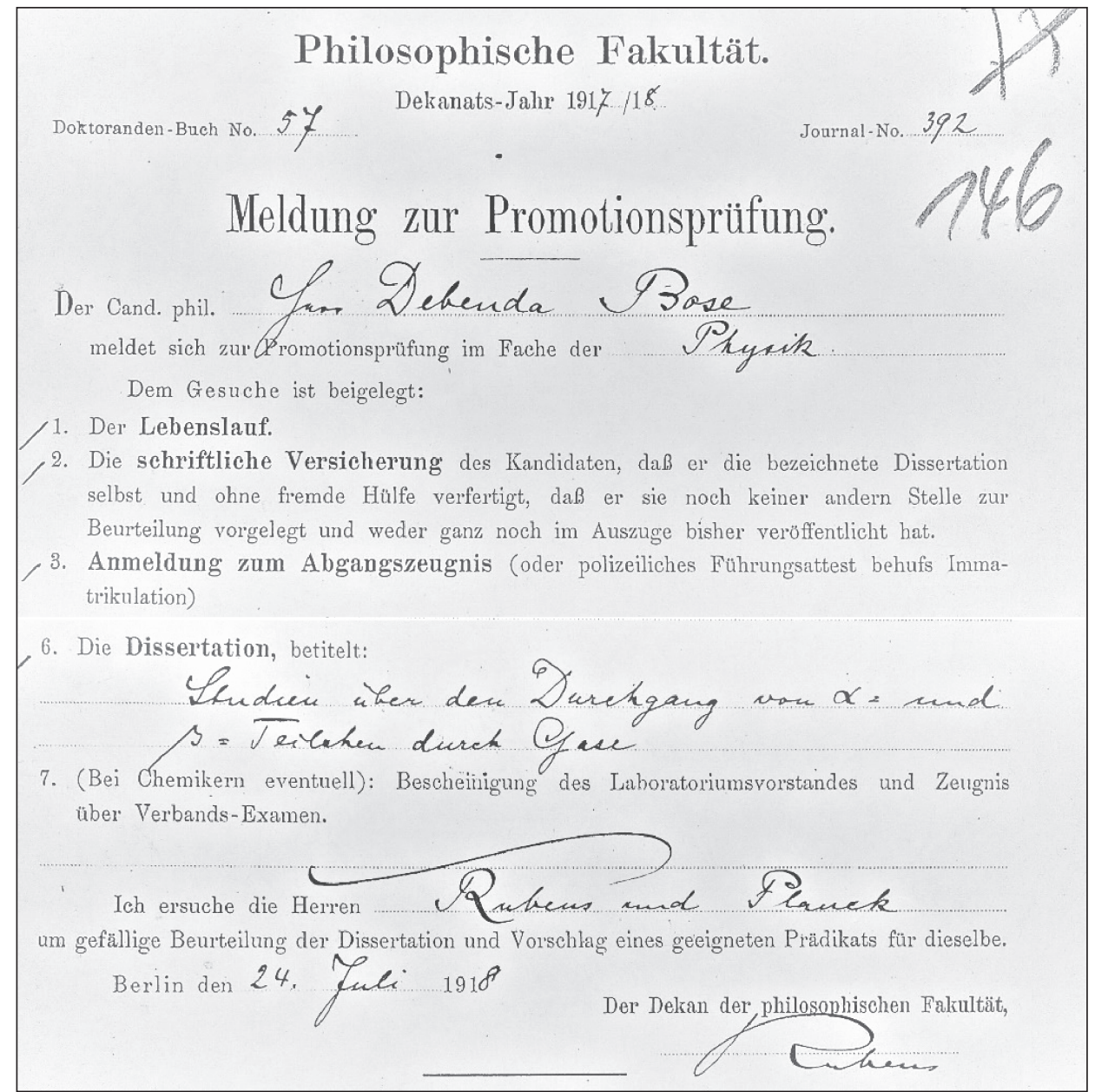

Fig. 3. A part of D M Bose's PhD registration certificate. (Credit: Humboldt University, Berlin).

in literature was referred to as 'Bose effect's; c) initiating particle physics in India. His better known work was on cosmic rays researches, in particular, detection of mesotrons or mesons with photographic emulsions, which he did in association of Bibha Chaudhuri'; and d) Physico-Chemical Hypothesis, in the field of plant physiology.

D M Bose avoided limelight. In 1938, after becoming the Director of the Bose Institute, he invested most of his time in improving the Institute. Due to his efforts new fields of research were initiated. These details are explored elsewhere (Singh \& Roy, 2017; Bose, 1958).
Due to his modest ways of living, and shun of publicity, his scientific achievements remained unnoticed by the scientific community. In the Economic Times of May 31, 1994, Rajesh Kochhar wrote that at the $70^{\text {th }}$ birthday of S N Bose, one of the speakers, D M Bose referred to an invitation from Como (Italy). He was interrupted by $\mathrm{S} \mathrm{N}$ Bose. The latter was of the opinion that the invitation was for him. According to other participant, such discussion never occurred. ${ }^{7}$ The fact is in 1927, D M Bose attended the Como Conference due to an invitation (Roy \& Singh, 2017, pp. 37-46; Singh, 2010).

\footnotetext{
${ }^{6}$ For more detail, see, Roy \& Singh, 2015.

${ }^{7} \mathrm{I}$ am thankful to the anonymous referee for this information.
} 
D M Bose occupied many important positions, such as Ghosh Professor (1919-1934), and Palit Professor of Physics (1935-1938) at the University of Calcutta. In 1927, he was President of the Physics Section of Indian Science Congress Association. He was also Founder Member of Indian Physical Society; and Life Member of the Indian Association for the Cultivation of Sciences. He was Foundation Fellow of the Indian National Science Academy (INSA, 1985, p. 104); General President, Indian Science Congress (1952-1953); Vice President - INSA, 1953-1956, 1960-1961); first Editor of the journal Indian Journal of History of Science (1966-1974), Editor-in-Chief of the journal Science and Culture (1965-1975) and of the book A Concise History of Science in India, Indian National Science Academy, New Delhi, 1971 (Singh, 2016b, pp. 5-8).

\section{Conclusion}

In the forgoing discussion a few aspects of B B Ray's, D M Bose's and S K Mitra's lives and scientific works have been presented. All the three, by one way or other, influenced the development of physics in India by establishing different fields of researches. In spite of that they did not receive the due appreciation by historians and their colleagues. More research work is needed to explore the causes in terms of social-, cultural- and political contexts.

\section{ACKNowledgement}

I am indeed thankful to S C Roy (Science and Culture); Maria Asp Dahlbäck and Karl Grandin (Center for History of Science, the Royal Swedish Academy of Sciences, Stockholm); Joanna Hopkins (Archive, Royal Society of London); Finn Aaserud, Felicity Pors and Lis Rasmussen (Niels Bohr Archive, Copenhagen); Subrata Roy (Central Silk Technological Research Institute, Bangalore); Debabrata Ray, (National Education Commission, Bengal); Philosophical Archive (University of Konstanz, Germany) for support and supplying important source materials on Roy, Mitra and Bose.

Thank are also due to Michael Komorek, Head of the Research Group - Physics Didactics and History of Science, for providing research facilities.

\section{BiBLIOGRAPHY}

Anonymous, Determination of spectroscopic standard wavelengths in the short wave-length region, J. Chem. Soc. Abstr., 124 (1923):ii595-ii596.

Anonymous, Obituary - Professor Bidhubhusan Ray, Sci. Cult. 10 (1944-1945):111-112.

Anonymous, Prof. B B Ray, Nature, 154 (1944):818-819.

Bose, D M. Bose Institute - A short account of its research activities since 1917, Trans. Bose Res. Inst., 22 (1958): xvii-xxxviii.

Bose, D N. Ions and electrons in action, Sci. Cult., 76 (2010):510-517.

Burma, D P. J C Bose Medal Lecture 1980 - Bose Institute, Myself and Ribosome, Proc. Indian Nat. Sci. Acad., 47 (1981):17-25.

Chatterjee, S D. Debendra Mohan Bose (1885-1975), Biogr. Mem. Fellows INSA, 7 (1975):85-101.

D M Bose seventieth Birthday commemoration volume, Trans. Bose Res. Inst., 20 (1955): v-xi.

Das Gupta, M K. Reflections - Professor Sisir Kumar Mitra - As I remember him, Resonance, 5 (2000):92-99.

Fellows of the Indian National Science Academy 1935-1993, Vol. I, A-M, Biographical notes, Diamond jubilee compendium, Indian National Science Academy, New Delhi, 1994.

Ghosh, S N and Ghosh, A. Professor S K Mitra - His pioneering work on radio science, Curr. Sci., 68 (1995):1150-1159.

http://insaindia.res.in/deceaseddetail.php?id=N350654, Oct. 31,2016

Mahanti, S. Sisir Kumar Mitra - A pioneer in radio physics, Dream, 2047 (2003): 29-34.

Minutes of the syndicate 1923 - Part I \& II, Calcutta University Press, Calcutta, 1923.

Mitra, S K. On a new geometrical theory of the diffractionfigures observed in the heliometer, Proc. IACS., 6 (1920): 1-18. 
Mitra, S K. Determination of spectroscopic standard wavelengths in the short wave-length region, Ann. Physique, 19 (1923): 315-339.

Mukherji, P. Debendra Mohan Bose (1885-1975) - A prominent physicist of India, Curr. Sci., 109 (2015): 2322-2324.

Ramanathan, K R. Sisir Kumar Mitra Memorial Lecture, Proc. Nat. Inst. Sci. (India), 35 (1967): 268-281.

Ratcliffe, J A. Sisir Kumar Mitra (1890-1963), Bio. Mem. Fell. R. Soc. Lond., 10 (1964): 221-228.

Roy, S C. D.M. Bose - A scientist incognito, Sci. Cult., 76 (2010): 491-493.

Roy, S C and Singh, R. D M Bose and cosmic ray research, Indian Journal of History of Science, 50.3 (2015):438455.

Roy, S C and Singh, R. D M Bose - A scientist incognito, Bose Institute Publication, Kolkata, 2017.

Science in India - 50 years of the Academy, Indian National Science Academy, New Delhi, 1985.

Sen, S N. Late Dr. Bidhu Bhusan Ray - A life devoted to science, The Sunday Hindustan Standard, Aug. 13, 1944.

Singh, R and Riess, F. Bidhu Bhushan Ray and his Western connections, Sci. Cult., 66 (2001):177-181.

Singh, R and Roy, S C. Coming of age - Bose Institute under D M Bose, Sci. Cult., 83 (2017): 364-379.
Singh, R. Celebrating $125^{\text {th }}$ birth anniversary of DM Bose Invitation to the Como conference, Sci. Cult., 76 (2010): 494-501.

Singh, R. Nobel Prize Nominator Sisir Kumar Mitra F.R.S. - His scientific work in international context, Shaker Verlag, Aachen, 2014.

Singh, R. Chemistry and Physics Nobel Prizes - India's Contribution, Shaker Verlag, Aachen, 2016.

Singh, R. D M Bose - His Scientific Work in International Context, Shaker Verlag, Aachen, 2016.

Singh, R. Bidhu Bhushan Ray - A Pioneer of X-ray Spectroscopy, Shaker Verlag, Aachen, 2017.

Singh, R. B B Ray and Controversy over the Spectral Raman-lines, Indian Journal of History of Science 52.3 (2017): 343-350.

Singh, R. Sisir Kumar Mitra, Scientific achievements and the Fellow of the Royal Society London, Indian Journal of History of Science, 52.4 (2017): 407-419.

Singh, R. B B Ray under the influence of C V Raman and M N Saha, Sci. Cult., 83 (2017): 84-91.

Singh, R. Netaji Subhas Chandra Bose, Bidhu Bhushan Ray and 'Science and Culture' - History of Science vs. Political History, Sci. Cult. (forthcoming).

van Bokhoven, J A and Lamberti, C. (Eds.). X-ray absorption and X-ray emission spectroscopy - Theory and applications, John Wiley \& Sons, Ltd., West Sussex, 2016. 